\title{
Clinical characteristics and treatment outcomes of patients with Brugada syndrome in northeastern Thailand
}

\author{
Pattarapong Makarawate ${ }^{1}$, MD, Narumol Chaosuwannakit ${ }^{2}$, MD, Suda Vannaprasaht ${ }^{3}$,MD, \\ Wichittra Tassaneeyaku/ ${ }^{3,4}$, PhD, Kittisak Sawanyawisuth ${ }^{1,5}$, MD, PhD
}

INTRODUCTION Brugada syndrome $(\mathrm{BrS})$ is a common genetic cause of sudden cardiac arrest (SCA) due to polymorphic ventricular tachycardia and ventricular fibrillation. The current recommended therapy for high-risk BrS patients is the use of an implantable cardioverter defibrillator (ICD). The present study aimed to report the clinical characteristics and treatment outcomes of BrS patients in northeastern Thailand.

METHODS Patients who were diagnosed with BrS or had a Brugada electrocardiogram (ECG) between 2005 and 2012 at Khon Kaen University's hospitals were enrolled in the present study. Patients' clinical characteristics, ECG type, laboratory results and treatment were reviewed.

RESULTS A total of 90 eligible patients were enrolled. Of these, 79 (87.8\%) patients were symptomatic - 65 (82.3\%) had documented SCA and 14 (17.7\%) had unexplained syncope. The remaining 11 (12.2\%) patients were asymptomatic with Brugada ECG. A majority of the patients enrolled were born in northeastern Thailand. The mean age of the symptomatic patients was $44.49 \pm 8.55$ years. Among the symptomatic patients, a majority were male $(\mathrm{n}=77,97.5 \%)$ and $23(29.1 \%)$ patients had a family history of SCA. Almost all BrS patients who were symptomatic (96.2\%) received ICD treatment for secondary prevention. The number of patients who received appropriate ICD therapy was 4.2 times of those who received inappropriate shocks. Only 3 (3.8\%) symptomatic BrS patients refused ICD treatment.

CONCLUSION Clinical characteristics did not distinguish between symptomatic BrS patients and asymptomatic patients with Brugada ECGs. The clinical characteristics and treatment outcomes for the symptomatic BrS patients with SCA and unexplained syncope were similar. Among the BrS patients implanted with secondary prevention ICD in Northeastern Thailand, nearly one-third had received appropriate ICD therapy, far exceeding the incidence of device-related complications and inappropriate therapy.

Keywords: Brugada syndrome, implantable cardioverter defibrillator, outcomes, treatment

\section{INTRODUCTION}

Brugada syndrome $(\mathrm{BrS})$ is characterised by ST segment elevation (coved type) in the right precordial leads on electrocardiography (ECG), and sudden cardiac death in patients with a structurally normal heart. In Thailand, it was discovered that many post-resuscitated patients had a Brugada ECG and a family history of sudden unexplained death syndrome (SUDS). (1) BrS typically manifests during adulthood, with sudden death occurring at a mean age of $41 \pm 15$ years, and ventricular tachycardia (VT) or ventricular fibrillation (VF occurring predominantly at rest or during sleep. ${ }^{(1-5)} \mathrm{A}$ Brugada ECG or a coved-type ECG pattern is key in the diagnosis of BrS. This ECG pattern may be found in persons with a family history of SUDS or in asymptomatic persons. Several methods, such as a postexercise examination, medications (e.g. sodium channel-blocking agents) and the use of high intercostal leads, can be used to unmask the Brugada ECG. ${ }^{(6,7)}$ Early detection of Brugada ECG may indicate risks of developing SUDS or sudden cardiac arrest (SCA).
For symptomatic $\mathrm{BrS}$ patients, treatment with an implantable cardioverter defibrillator (ICD) is more effective than the use of antiarrhythmic drugs. ${ }^{(8)}$ Currently, ICD implantation is the standard treatment for secondary prevention in symptomatic BrS patients, including aborted SCA and syncope of unknown origin. ${ }^{(9)}$ Data on the clinical characteristics and treatment outcomes of patients with $\mathrm{BrS}$ and asymptomatic patients with Brugada ECG is still limited. We therefore conducted a study on a large series of patients with BrS from northeastern Thailand, an area endemic for $\mathrm{BrS}$, and herein report our findings.

\section{METHODS}

Patients diagnosed with BrS or had a Brugada ECG between 2005 and 2012 at the Queen Sirikit Heart Center of the Northeast and Srinagarind Hospital, Khon Kaen University, Thailand, were enrolled in the present study. All eligible patients were classified into two groups - symptomatic BrS patients and asymptomatic patients with a Brugada ECG. BrS was diagnosed clinically in patients presenting with SCA or

\footnotetext{
${ }^{1}$ Department of Medicine, ${ }^{2}$ Department of Radiology, ${ }^{3}$ Department of Pharmacology, Faculty of Medicine, ${ }^{4}$ Research and Diagnostic Center for Emerging Infectious Diseases, ${ }^{5}$ The Research and Training Center for Enhancing Quality of Life of Working-Age People, Khon Kaen University, Khon Kaen, Thailand Correspondence: Dr Kittisak Sawanyawisuth, Associate Professor, Department of Medicine, Faculty of Medicine, Khon Kaen University, Khon Kaen 40002, Thailand. kittisak@kku.ac.th
} 
Table I. Demographic and clinical characteristics of the study population $(n=90)$.

\begin{tabular}{|c|c|c|c|}
\hline \multirow[t]{2}{*}{ Characteristic } & \multicolumn{2}{|c|}{ No. (\%) } & \multirow[t]{2}{*}{ p-value } \\
\hline & $\begin{array}{l}\text { Symptomatic BrS } \\
(n=79)\end{array}$ & $\begin{array}{l}\text { Asymptomatic with Brugada ECG } \\
\qquad(\mathrm{n}=11)\end{array}$ & \\
\hline Age* (yrs) $^{*}$ & $44.49 \pm 8.55$ & $46.36 \pm 10.94$ & 0.384 \\
\hline Male gender & $77(97.5)$ & $11(100.0)$ & 1.000 \\
\hline Familial history of sudden death & $23(29.1)$ & $5(45.5)$ & 0.307 \\
\hline Spontaneous type I ECG & $60(75.9)$ & $10(90.9)$ & 0.444 \\
\hline Type 1 ECG with high intercostal leads & $16(20.3)$ & $1(9.1)$ & 0.683 \\
\hline Type 1 ECG after sodium channel blockers & $3(3.8)$ & $0(0)$ & 1.000 \\
\hline Residency region in Thailand & & & 0.594 \\
\hline Northeastern & $70(88.6)$ & $11(100.0)$ & \\
\hline Northern & $6(7.6)$ & $0(0)$ & \\
\hline Southern & $1(1.3)$ & $0(0)$ & \\
\hline Central & $2(2.5)$ & $0(0)$ & \\
\hline
\end{tabular}

${ }^{*}$ Data is presented as mean \pm standard deviation. BrS: Brugada syndrome; ECG: electrocardiogram

syncope, and any one of the following forms of evidence: (a) documented VT or VF; (b) a family history of sudden cardiac death at $<45$ years of age; (c) the presence of Brugada ECG in family members; (d) unexplained syncope; (e) a history of nocturnal agonal respiration with spontaneous type 1 Brugada ECG; or (f) type 1 Brugada ECG by high intercostal leads or with the administration of sodium channel blockers. Type 1 Brugada ECG was defined as a prominent coved ST segment elevation (i.e. $>2 \mathrm{~mm}$ ) followed by a negative T wave. ${ }^{(10-12)}$ Patients who were asymptomatic and had a Brugada ECG were categorised into the asymptomatic group. The following clinical data were collected: age at diagnosis, gender, birth place, family history of SCA, type of Brugada ECG at diagnosis, results of pharmacological testing or use of high intercostal leads (for unmasking coved-type ECG pattern), circumstances of diagnosis (e.g. SCA or syncope), indication for ICD implantation, and outcomes of treatment.

Data was expressed as either mean \pm standard deviation or number (percentage). The clinical data of the symptomatic and asymptomatic groups were studied and compared. For further analysis, symptomatic BrS patients were classified according to their presenting symptoms (i.e. SCA or unexplained syncope), and their clinical characteristics and outcomes after ICD treatment were compared according to their presenting symptoms. Unpaired t-tests or Mann-Whitney $U$ tests were used to test for significant differences between continuous variables, while chi-square or Fisher's exact tests were used for categorical variables. A p-value of $<0.05$ was considered statistically significant. All patients were informed verbally and in writing about the study procedure and purpose. The study protocol was approved by the Khon Kaen Ethics Committee for Human Research, Khon Kaen University, Thailand (HE 51024, HE 551129).

\section{RESULTS}

A total of 90 eligible patients (mean age $46.00 \pm 5.64$ years) were enrolled during the study period. The demographic and clinical characteristics of the study population are summarised in Table I. 79 (87.8\%) patients presented with symptoms of BrS. Of these, SCA was documented in 65 (82.3\%) patients and syncope of unknown origin was found in $14(17.7 \%)$ patients. There were $11(12.2 \%)$ asymptomatic patients with a Brugada ECG; 5 of the 11 (45.5\%) patients had a family history of SCA. A majority of symptomatic BrS patients were male $(\mathrm{n}=77,97.5 \%)$, and born and raised in northeastern Thailand ( $\mathrm{n}=70,88.6 \%$ ). A family history of SCA was found in 23 of the 79 (29.1\%) symptomatic BrS patients. A spontaneous coved-type Brugada ECG was found in 60 of the 79 (75.9\%) patients at baseline diagnosis, while high intercostal leads revealed the diagnosis of a coved-type Brugada ECG in 16 of the $19(84.2 \%)$ symptomatic BrS patients who did not show a typical coved-type Brugada ECG at diagnosis. All factors, including age, gender, family history of sudden death, ECG type, and area of residence, were comparable between the symptomatic BrS patients and the asymptomatic patients with Brugada ECG (Table I).

No significant difference in demographic and clinical characteristics were found between symptomatic $\mathrm{BrS}$ patients presenting with SCA and those with unexplained syncope (Table II). The number of symptomatic BrS patients who received ICD implantation, received appropriate ICD treatment, had complications of ICD, and died are shown in Table III. A single-chamber ICD was implanted in 76 (96.2\%) symptomatic BrS patients - $64(84.2 \%)$ with SCA and 12 (15.8\%) with unexplained syncope. The ICD was set to $1 \mathrm{VF}$ zone, with a detection zone rate of more than $180 \mathrm{bpm}$ and a monitor VT zone rate of 150-180 bpm. Three symptomatic BrS patients refused ICD treatment (one SCA, two unexplained syncope); these patients were found to be free of symptoms in the three years of follow-up.

None of the patients was lost to follow-up during the study period. The average follow-up time of the 79 symptomatic $\mathrm{BrS}$ patients was 4.80 years. Of the 76 symptomatic $\mathrm{BrS}$ patients who had ICD treatment, 25 (32.9\%) received appropriate shock therapy. Of these 25 patients, 21 were in the post-SCA group and 4 were in the unexplained syncope group. All 
Table II. Demographic and clinical characteristics of symptomatic patients with Brugada syndrome, according to clinical presentation $(n=79)$.

\begin{tabular}{|c|c|c|c|}
\hline \multirow[t]{2}{*}{ Characteristic } & \multicolumn{2}{|c|}{ No. (\%) } & \multirow[t]{2}{*}{ p-value } \\
\hline & Sudden cardiac arrest $(n=65)$ & Unexplained syncope $(n=14)$ & \\
\hline Age* (yrs) $^{*}$ & $44.17 \pm 8.80$ & $46.00 \pm 7.37$ & 0.322 \\
\hline Male gender & $64(98.5)$ & $13(92.9)$ & 0.325 \\
\hline Familial history of sudden death & $17(26.2)$ & $6(42.9)$ & 0.330 \\
\hline Spontaneous type I ECG & $48(73.8)$ & $12(85.7)$ & 0.498 \\
\hline Type 1 ECG with high intercostal leads & $14(21.5)$ & $2(14.3)$ & 0.723 \\
\hline Type 1 ECG after sodium channel blockers & $3(4.6)$ & $0(0)$ & 1.000 \\
\hline Residency region in Thailand & & & 0.657 \\
\hline Northeastern & $58(89.2)$ & $12(85.7)$ & \\
\hline Northern & $4(6.2)$ & $2(14.3)$ & \\
\hline Southern & $1(1.5)$ & $0(0)$ & \\
\hline Central & $2(3.1)$ & $0(0)$ & \\
\hline
\end{tabular}

Data is presented as mean \pm standard deviation. ECG: electrocardiogram

Table III. Treatment and treatment outcomes of symptomatic patients with Brugada syndrome, according to clinical presentation $(n=79)$.

\begin{tabular}{lccc}
\hline Parameter & \multicolumn{2}{c}{ No. (\%) } & p-value \\
\cline { 2 - 3 } & Sudden cardiac arrest (n= 65) & Unexplained syncope (n = 14) & 0.079 \\
\hline ICD implantation & $64(98.5)$ & $12(85.7)$ & 1.000 \\
Appropriate ICD treatment & $21(32.3)$ & $4(28.6)$ & 0.102 \\
Complication after ICD treatment & $4(6.2)$ & $3(21.4)$ & 1.000 \\
Death & $0(0)$ & $0(0)$ & \\
\hline ICD: implantable cardioverter defibrillator & & &
\end{tabular}

appropriate shock therapies were administered due to detected VF. Most patients $(n=17,68.0 \%$ ) received the appropriate shock therapy at between 8:00 pm and 6:00 am, while the remaining patients $(\mathrm{n}=8,32.0 \%$ ) received it between 3:00 pm and 7:00 pm. The average follow-up time of all 11 asymptomatic patients with Brugada ECG was 4.82 years. During the follow-up period, none of the asymptomatic patients with Brugada ECG had SCA, syncope or serious cardiac arrhythmia.

Complications after ICD treatment occurred in 7 (9.2\%) patients -6 patients received inappropriate ICD shock and 1 patient developed an ICD infection, which necessitated the removal of the ICD and its subsequent reimplantation after the infection was eradicated. Reasons for inappropriate shock were lead fracture (3 patients) and supraventricular arrhythmia (1 paroxysmal supraventricular tachycardia, 1 atrial fibrillation and 1 sinus tachycardia).

\section{DISCUSSION}

In the present study, we found that the demographic and clinical characteristics (i.e. age, gender, family history of sudden death, ECG type and area of residence) of symptomatic $\mathrm{BrS}$ patients and asymptomatic patients with Brugada ECG did not differ significantly (Table I). Almost all of the patients were male and resided in northeastern Thailand. Asymptomatic patients with Brugada ECG had a higher ratio of family history of sudden death. Family members of BrS patients are urged to undergo a 12-channel ECG test with high intercostal leads or medications to detect Brugada ECG. The prevalence of a family history of SCA in the symptomatic $\mathrm{BrS}$ patients in our study was higher than that reported in a study from Europe (29.1\% vs 9.1\%). ${ }^{(13)}$ In the present study, $20.3 \%$ of the Brugada ECGs in symptomatic $\mathrm{BrS}$ patients was unmasked with the use of high intercostal leads. This finding is compatible with a previous report by Sangwatanaroj et $\mathrm{al}^{\left({ }^{(5)}\right.}$ In which, the authors recommended that family members of victims of SUDS be screened using ECG with high intercostal leads. ${ }^{(5)}$ However, further studies are needed to justify such a screening exercise.

The clinical characteristics and treatment outcomes of symptomatic BrS patients who presented with SCA and unexplained syncope were comparable in the present study (Tables II \& III). Almost all of the symptomatic BrS patients underwent ICD implantation (76/79, 96.2\%). Of these 76 patients, $25(32.8 \%)$ received appropriate shock therapy. This number is 4.2 times the number of patients who received inappropriate shocks $(n=6)$. In contrast, a study by Sacher et $\mathrm{al}^{(14)}$ found that inappropriate shocks occurred 2.5 times more frequently than appropriate shock therapy. This difference may be due to differences in the study population of the two studies; the present study had more patients with documented SCA than the study by Sacher et al (82.3\% vs $8 \%)$.

In our study, at least $31.6 \%$ (25/79) of the symptomatic BrS patients reaped the benefits of ICD implantation in preventing further VT/VF. The shock therapies occurred mainly during the 
night or while the patients were sleeping $(68.0 \%)$, similar to previous reports. ${ }^{(11,12)}$ The three symptomatic $\mathrm{BrS}$ patients who refused ICD implantation and were treated with amiodarone instead had no recurrence of symptoms in the three years of follow-up.

We found a family history of SCA in 5 (45.5\%) asymptomatic patients with Brugada ECG (Table I). All of the 11 asymptomatic patients with Brugada ECG did not experience SCAs, syncopes or serious arrhythmias during a period of follow-up that lasted for approximately five years. This finding suggests that asymptomatic patients with Brugada ECG may have a low risk for SCA or serious cardiac arrhythmias and thus do not require treatment with ICD or antiarrhythmic agents. This, however, needs to be validated with further studies.

To conclude, demographic and clinical characteristics did not distinguish between symptomatic BrS patients and asymptomatic patients with Brugada ECG. Among symptomatic $\mathrm{BrS}$ patients who presented with either SCA or unexplained syncope, the demographic and clinical characteristics, as well as treatment outcomes, were similar. Nearly one-third of the $\mathrm{BrS}$ patients in northeastern Thailand who were implanted with secondary prevention ICD received appropriate ICD therapy, far exceeding the incidence of device-related complications and inappropriate therapy.

\section{ACKNOWLEDGEMENTS}

The authors would like to thank Professor James A Will, University of Wisconsin, USA, for his kind assistance in the English-language editing of this article. This study was supported by TRF grants from the Senior Research Scholar Grant, Thailand Research Fund (grant number RTA5580004), and the Higher Education Research Promotion and National Research
University Project of Thailand, Office of the Higher Education Commission, Thailand, through the Health Cluster (SHeP-GMS), Khon Kaen University.

\section{REFERENCES}

1. Tatsanavivat $P$, Chirawatkul A, Klungboonkrong $V$, et al. Sudden and unexplained deaths in sleep (Laitai) of young men in rural northeastern Thailand. Int J Epidemiol 1992; 21:904-10.

2. Sung RJ, Kuo CT, Wu SN, et al. Sudden cardiac death syndrome: age, gender, ethnicity and genetics. Acta Cardiol Sin 2008; 24:65-74.

3. Nademanee K, Veerakul K, Nimmannit S, et al. Arrhythmogenic marker for the sudden unexplained death syndrome in Thai men. Circulation 1997; 96:2595-600.

4. Gussak I, Antzelevitch C, Bjerregaard P, Towbin JA, Chaitman BR. The Brugada syndrome: clinical, electrophysiologic and genetic aspects. J Am Coll Cardiol 1999; 33:5-15.

5. Sangwatanaroj S, Prechawat S, Sunsaneewitayakul B, et al. Right ventricular electrocardiographic leads for detection of Brugada syndrome in sudden unexplained death syndrome survivors and their relatives. Clin Cardiol 2001; 24:776-81.

6. Makarawate P, Sangwatanaroj S. Effect of high glycemic index diets on ST segment elevation in Brugada syndrome. Asean Heart J 2008; 15:57-62.

7. Sangwatanaroj S, Prechawat S, Sunsaneewitayakul B, et al. New electrocardiographic leads and the procainamide test for the detection of the Brugada sign in sudden unexplained death syndrome survivors and their relatives. Eur Heart J 2001; 22:2290-6.

8. Nademanee K, Veerakul G, Mower M, et al. Defibrillator versus beta blockers for unexplained death in Thailand (DEBUT): a randomized clinical trial. Circulation 2003; 107:2221-6.

9. Brugada J, Brugada R, Brugada P. Pharmacological and device approach to therapy of inherited cardiac disease associated with cardiac arrhythmias and sudden death. J Electrocardiol 2000; 33 suppl 1:41-7.

10. Antzelevitch C. Brugada syndrome. Pacing Clin Electrophysiol 2006; 29:1130-59.

11. Brugada $P$, Benito B, Brugada R, Brugada J. Brugada syndrome: update 2009. Hellenic J Cardiol 2009; 50:352-72.

12. Berne P, Brugada J. Brugada syndrome 2012. Circulation 2012; 76:1563-71.

13. Sarkozy A, Sorgente A, Boussy T, et al. The value of family history of sudden death in patients with diagnosis type 1 Brugada ECG pattern. Eur Heart J 2011; 32:2153-60.

14. Sacher F, Probst V, lesaka Y, et al. Outcome after implantation of a cardioverter-defibrillator in patients with Brugada syndrome: a multicenter study. Circulation 2006; 114:2317-24. 